\section{Research advance directives}

Stocking et al. found that persons with mild to moderate dementia may still be able to complete an unofficial research advance directive and choose which types of research they wish to enroll in at some future time. Although 83\% would defer that decision to their proxies at enrollment time, $17 \%$ would not.

see page 1361

\section{Cognitive performance and decisional capacity in dementia}

The capacity to make treatment decisions is impaired in dementia, but the underlying cognitive processes are unclear. Gurrera et al. administered neuropsychological tests to 88 individuals with mild to moderate dementia. Test performance accounted for 10 to $78 \%$ of the variance in decisional abilities; most abilities had distinct neuropsychological predictor $(\beta)$ profiles.

see page 1367

The editorial by Kim and Kieburtz on these two articles notes that current federal regulations allows a "legally authorized representative" to give permission on behalf of another person to participate in research, but defer to the states to define legally authorized representative. A policy consensus on proxy consent for research has been elusive. One such solution-advance directives for research, completed prior to onset of dementia-is unlikely to play a major role because only a minority of persons complete an advance directive even for treatment decisions, and fewer still will contemplate ahead of time the unpleasant possibility of developing dementia and participating in research. The idea that persons with dementia may be able to legitimately delegate authority for research decision-making is intriguing. While further empirical research may not support the concept of a relatively preserved capacity to appoint a proxy, there is a need to have more empirically informed policy discussions.

see page 1298

\section{Lacunar stroke and risk of further events}

Sacco et al. report data from a population-based study showing that patients with lacunar stroke in the long term have a risk of death and of stroke recurrence similar to that of patients with nonlacunar stroke.

\section{Retinal vessels and stroke}

Ikram et al. showed in a large prospective populationbased study that larger retinal venular diameters are associated with an increased risk of stroke and cerebral infarction.

see page 1339

\section{Genetic study of APOE variation in MS}

Burwick et al. report data from a large number of patients with MS and their families genotyped for APOE epsilon polymorphisms. Their findings did not support a prominent role for APOE in MS.

see page 1373

\section{Lamotrigine vs carbamazepine for epilepsy}

In a meta-analysis of trials comparing carbamazepine and lamotrigine for epilepsy, Gamble et al. found that overall, lamotrigine is better tolerated, but trends favored carbamazepine for seizure control.

see page 1310

The accompanying editorial by French and Kryscio notes that this type of head-to-head comparison should determine whether "newer drugs are better than old," or at least not worse, which is the question on the minds of clinicians as well as individuals responsible for making cost decisions. This meta-analysis illustrates that things are not as simple as they would appear. The primary outcome of the analysis showed that lamotrigine is better tolerated than carbamazepine, a significant and clinically important finding. However, it also suggested that carbamazepine may have a clinically important advantage in time to remission and time to first seizure. The fact that the important AED qualities of tolerability and efficacy go in opposite directions makes it difficult to identify the better therapy. They ask if the trials analyzed by Gambel et al. were designed to use each drug to its maximal advantage. They suggest not. They propose that an unbiased panel of individuals should have input into the design of an active control study since all potential external validity issues need to be decided in advance. 


\section{Neuroanatomic correlates of stroke-related myocardial injury}

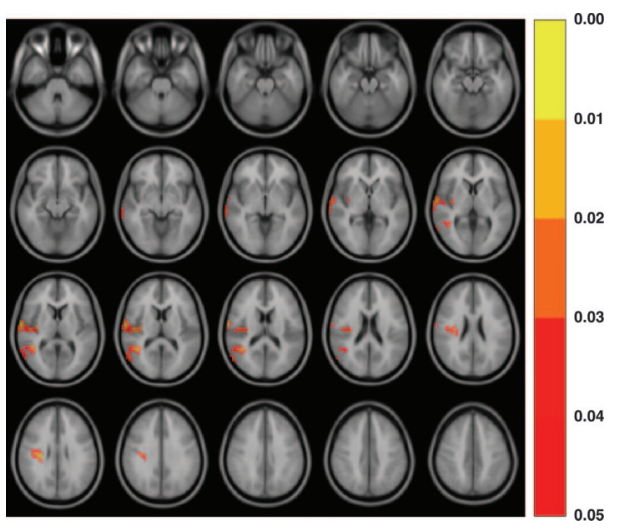

Map of cerebral sites associated with troponin-T elevation.

Ay et al. studied patients with acute ischemic stroke in whom serum cardiac troponin-T elevation occurred in the absence of any apparent cause. Using a method free from the bias of an a priori hypothesis as to any specific location, the authors produced a map of brain regions, which when infarcted, were associated with myocardial injury. This map revealed that infarctions in the right posterior-, superior-, and medial-insula and the right inferior parietal lobule were associated with elevated serum troponin-T level indicative of myocardial injury.

see page 1325

\section{Troponin and mortality in intracerebral hemorrhage}

Elevated cardiac troponin levels in patients with ischemic stroke and subarachnoid hemorrhage are thought to be due to sympathetic activation and have been associated with higher mortality. Hays and Diringer now report that this phenomenon also occurs in intracerebral hemorrhage.

see page 1330

The editorial by Cheshire and Saper on these two articles notes that these two studies further tighten the link between the insular cortex and the heart. Cardiac troponin elevations suggesting myocardial injury occurred following both ischemic stroke and intracerebral hemorrhage. The frequent evidence of myocardial injury suggests that the pathophysiology may be similar to that in experimental animals in which insular cortex lesions unleash sympathetic neural activation leading to cardiac myocytolysis, so-called contraction band necrosis. While measuring cardiac troponin levels may not yet be appropriate for all stroke patients, it is possible that patients with insular strokes are at high risk of neurogenic cardiac effects. Furthermore, if the cardiotoxic effect of stroke is as common as these studies suggest, it may be prudent to consider therapy that protects the heart in patients presenting with right insular infarctions.

see page 1296

\section{Natural history risk of untreated brain arteriovenous malformations (AVMs)}
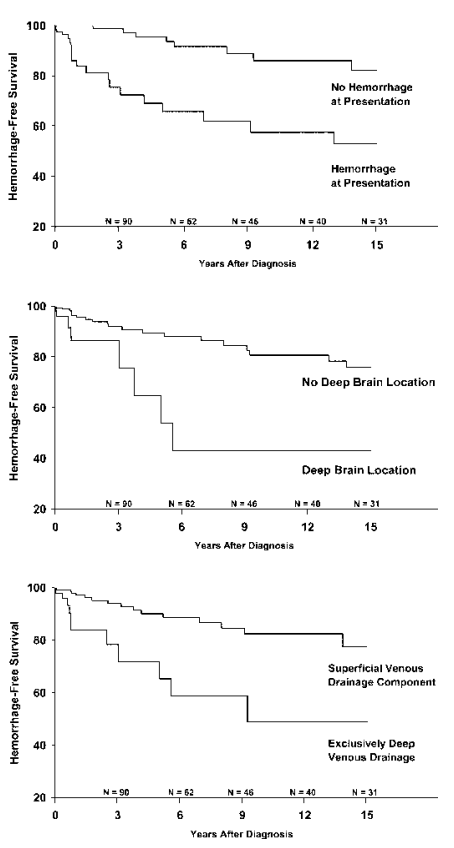

Stapf et al. prospectively studied 622 untreated patients with arteriovenous malformations. Hemorrhagic presentation (HR 5.38), deep AVM location (HR 3.25), exclusive deep venous drainage (HR 2.39), and age (HR 1.05) constitute independent predictors for AVM rupture on follow-up. Average annual hemorrhage rate was only $0.9 \%$ for patients with AVM without these risk factors.

see page 1350 


\title{
Neurology
}

\author{
May 9 Highlights \\ Neurology 2006;66;1292-1293 \\ DOI 10.1212/01.wnl.0000219225.64888.2b
}

\section{This information is current as of May 8, 2006}

\section{Updated Information \&}

Services

Permissions \& Licensing

Reprints including high resolution figures, can be found at: http://n.neurology.org/content/66/9/1292.full

Information about reproducing this article in parts (figures,tables) or in its entirety can be found online at:

http://www.neurology.org/about/about_the_journal\#permissions

Information about ordering reprints can be found online:

http://n.neurology.org/subscribers/advertise

Neurology ${ }^{\circledR}$ is the official journal of the American Academy of Neurology. Published continuously since 1951, it is now a weekly with 48 issues per year. Copyright. All rights reserved. Print ISSN: 0028-3878. Online ISSN: 1526-632X.

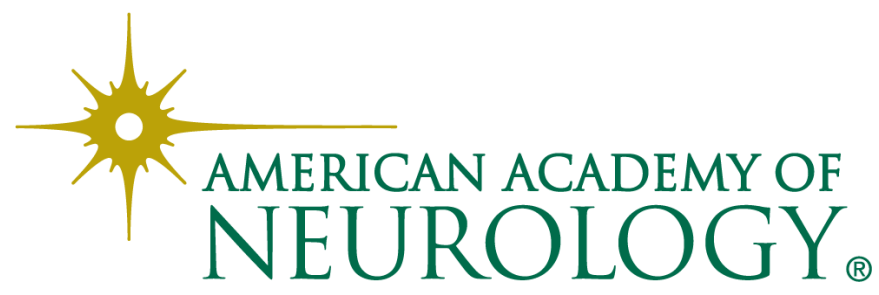

